\title{
Experimental and Modelling Study of Gravity Drainage in a Three-Block System
}

\author{
Hamidreza Erfani ${ }^{1,2}$ - Abtin Karimi Malekabadi ${ }^{3} \cdot$ Mohammad Hossein Ghazanfari $^{2}$. \\ Behzad Rostami ${ }^{4}$ iD
}

Received: 7 April 2020 / Accepted: 24 November 2020 / Published online: 2 December 2020

(C) The Author(s) 2020

\begin{abstract}
Gravity drainage is known as the controlling mechanism of oil recovery in naturally fractured reservoirs. The efficiency of this mechanism is controlled by block-to-block interactions through capillary continuity and/or reinfiltration processes. In this study, at first, several free-fall gravity drainage experiments were conducted on a well-designed threeblock apparatus and the role of tilt angle, spacers' permeability, wettability and effective contact area (representing a different status of the block-to-block interactions between matrix blocks) on the recovery efficiency were investigated. Then, an experimental-based numerical model of free-fall gravity drainage process was developed, validated and used for monitoring the saturation profiles along with the matrix blocks. Results showed that gas wetting condition of horizontal fracture weakens the capillary continuity and in consequence decreases the recovery factor in comparison with the original liquid wetting condition. Moreover, higher spacers' permeability increases oil recovery at early times, while it decreases the ultimate recovery factor. Tilt angle from the vertical axis decreases recovery factor, due to greater connectivity of matrix blocks to vertical fracture and consequent channelling. Decreasing horizontal fracture aperture decreases recovery at early times but increases the ultimate recovery due to a greater extent of capillary continuity between the adjacent blocks. Well match observed between the numerical model results and the experimental data of oil recovery makes the COMSOL multiphysics model attractive for application in multi-blocks fractured systems considering block-to-block interactions. The findings of this research improve our understanding of the role of different fracture properties on the block-to-block interactions and how they change the ultimate recovery of a multiblock system.
\end{abstract}

Keywords Fractured reservoirs · Gravity drainage · Three-block system · Reinfiltration · Block-to-block interaction

\section{List of Symbols \\ $\mathbb{K} \quad$ Permeability tensor \\ B Bond number}

Hamidreza Erfani

hamidreza.erfanigahrooei@manchester.ac.uk

Extended author information available on the last page of the article 
$b \quad$ Fracture aperture

EOR Enhanced oil recovery

$g \quad$ Gravitational acceleration

$H \quad$ Block height

$K \quad$ Absolute permeability

$k_{r} \quad$ Relative permeability

$L \quad$ Length

NFR Naturally fracture reservoirs

$P \quad$ Pressure

$P_{\mathrm{dt}} \quad$ Fracture threshold pressure

$q \quad$ Flow rate

$r \quad$ Radius

$S \quad$ Saturation

$t \quad$ Time

$z \quad$ Elevation

\section{Greek Letters}

$\gamma \quad$ Fluid specific weight

$\lambda$ Pore size distribution index

$\mu \quad$ Viscosity

$\phi \quad$ Porosity

$\rho \quad$ Density

$\sigma \quad$ Interfacial tension

$\theta \quad$ Contact angle

\section{Subscripts}

$\alpha \quad$ Phase $\alpha$

c Capillary pressure

ct Threshold capillary pressure

e Effective saturation

f Fracture

g Gas/air phase

nw Non-wetting phase

o Oil phase

w Wetting phase

\section{Introduction}

Two-phase flow in fractured rocks is relevant to various application, ranging from geothermal energy (Babaei and Nick 2019; Erfani et al. 2019), groundwater contamination (Gleeson et al. 2009; Sebben et al. 2015; Aminnaji et al. 2019; Aljuboori et al. 2019; Dejam 2019), carbon capturing and storage (Emami-Meybodi et al. 2015; Babaei and Islam 2018; Erfani Gahrooei and Joonaki 2018; Dejam and Hassanzadeh 2018; Erfani et al. 2020; An et al. 2020b; Ershadnia et al. 2020), and soil salinization (Weisbrod and Dragila 2006) to enhanced hydrocarbon recovery (Farajzadeh et al. 2012; Rokhforouz and Akhlaghi Amiri 2017; Rokhforouz and Amiri 2018; Bakhshi et al. 2018; Rabbani et al. 2020a; Chen et al. 2020; An et al. 2020a). The complexity of the fluid flow in highly heterogeneous rocks 
is caused by the notion that the fluid flows in two different media, matrix block and fracture, which separates the fluids into flowing and stagnant regions due to property contrast (Erfani et al. 2019; Hasan et al. 2019; Soltanian et al. 2019; An et al. 2020c; Aziz et al. 2020; Rabbani et al. 2020b; Hasan et al. 2020). In recent decades, increase in worldwide oil demand has motivated many studies in this field, since naturally fractured reservoirs (NFR) hold about $60 \%$ of the total oil reserves in the world (Joonaki et al. 2016; Hakimov et al. 2019). Unique characteristics and heterogeneous nature of such reservoirs, in addition to different oil production mechanisms, make them relatively complicated to model and understand compared to conventional reservoirs.

Gravity drainage and spontaneous imbibition are important production mechanisms in NFRs (Saidi et al. 1979; Saidi 1983; Austad and Standnes 2003; Ameri et al. 2015; Aljuboori et al. 2019). Gravity drainage is a process in which the gravity acts as the main driving mechanism and the non-wetting phase (i.e., gas phase) pushes the oil out of the rock. It is an effective production mechanism in the gas-invaded zone due to a larger density difference. Spontaneous imbibition is usually effective in the water-invaded zone, where water is imbibed into the rock due to capillary forces and oil is produced to the fractures. Gravity drainage phenomenon can be classified into two main types: free-fall and forced gravity drainage. Free-fall gravity drainage is usually the major oil production mechanism in high permeability and thick reservoirs with a great drawdown pressure (Hagoort 1980; Chatzis and Ayatollahi 1993), while forced gravity drainage is a result of gas injection. Gravity and capillary are significant driving forces in gravity drainage. Along with wettability, the efficiency of the process is controlled by block-to-block interactions such as capillary continuity, which preserves the hydraulic continuity of the oil phase over fractures, and reinfiltration, occurring when the produced oil from a block enters the underneath matrix block due to gravity and/capillary forces (Dullien 2012). When the length of a liquid element is larger than the fracture aperture, the upper face of the lower porous matrix block will touch the liquid element, and consequently, a liquid bridge will be formed between the two blocks (Fig. 4). The existence of liquid bridges and reinfiltration provides the hydraulic continuity between the blocks and decreases the threshold height of the upper block. On the one hand, the bulk flow regime in the matrix and vertical fractures and, on the other hand, film flow in the horizontal fracture strongly influence the two-phase gas-oil flow in fractured oil reservoirs (Rostami et al. 2010).

Numerous experimental studies have been conducted on the gravity drainage mechanisms. The majority of these studies were performed on synthetic porous media such as coarse quartz grains sand packs with high permeability, so that the duration of each experiment, as well as the set-up height, can be adequately decreased (Moon and Bruce 2007; Zendehboudi et al. 2011; Saedi et al. 2015; Teng et al. 2016). Saidi et al. (1979) studied the interaction of matrix blocks and the presence of capillary continuity and reinfiltration between adjacent matrix blocks. Horizontal fracture aperture of $10 \mu \mathrm{m}$ was shown to be necessary to form stable liquid bridges in the horizontal fractures. Major results reported by researchers in this field could be classified into two general categories: first, increasing the model height and decreasing capillary pressure improve the process efficiency; second, the presence of a driving viscous force leads to earlier breakthrough and can improve the ultimate recovery factor (i.e., forced gravity drainage). Notably, lower recovery factor by increasing the gas injection rate is also reported after a critical gas injection rate (Rostami et al. 2010; Akhlaghi et al. 2012; Saedi et al. 2015; Zobeidi et al. 2016).

Firoozabadi and Markeset (1992) and Firoozabadi and Markeset (1994) used spacers of different thicknesses (up to $1.2 \mathrm{~mm}$ ) as horizontal fractures in a multi-stack block system. They showed the existence of capillary continuity in the fractures with the aperture of up to 
$1.2 \mathrm{~mm}$ and concluded that liquid bridges play an important role in controlling the gravity drainage mechanism. Effects of horizontal fracture properties on reinfiltration process have been examined by Sajadian et al. (1998), documenting that the reinfiltration rate significantly depends on the oil supply from the upper blocks as well as the spacer specifications. Zobeidi and Fassihi (2018) showed the importance of block-to-block interactions in miscible/immiscible gas-oil gravity drainage as well as solvent injection process.

Pore-scale experimental studies were also conducted by some researchers, which led to a better understanding of the governing parameters, such as horizontal fracture apertures, wettability and tilt angle, which were reported to be crucial for the stability and shape of the formed liquid bridges (Chatzis et al. 1988; Mashayekhizadeh et al. 2011; Bahari Moghaddam and Rasaei 2015; Khorshidian et al. 2018; Harimi et al. 2019).

Gravity drainage modelling can be categorized as analytical (Bech et al. 1991; Di Donato et al. 2006; Abbasi et al. 2018), numerical (Schechter and Guo 1996; Chow and Butler 1996; Saedi et al. 2015; Udoh et al. 2015; Dejam 2018; Rahmati and Rasaei 2019) and empirical-experimental (Li and Horne 2003; Zendehboudi et al. 2011) models. Some researchers investigated the gravity drainage mechanisms in tight rocks by introducing film flow phenomena (Nenniger and Storrow 1958). Pavone et al. (1989) assumed linear function for relative permeability and the logarithmic relation for capillary pressure and proposed an analytical model. Dejam et al. (2011) studied the impact of new capillary pressure and relative permeability functions to linearize the governing equations of gravity drainage. They calculated the saturation distribution and the production rate using inverse Laplace and Fourier transformation of the linearized equations. Dejam (2018) used a finegrid numerical model to study the influence of different fracture capillary pressure models on oil recovery and therefore the block-to-block interaction process in fractured porous media. They showed that the existence of fracture capillary pressure results in higher ultimate oil recovery factor. Aghabarari and Ghaedi (2019) introduced a new boundary condition for matrix blocks to include reinfiltration phenomena in commercial simulators.

In this research, free-fall gravity drainage in a three-block system is investigated experimentally and numerically. The main objective is scrutinizing the interactions of the adjacent blocks, particularly capillary continuity and reinfiltration phenomena, which control the efficiency of oil recovery by gravity drainage. For this purpose, a three-block experimental set-up was designed, including vertical and horizontal fractures. Then, an extensive set of experiments were conducted to examine the effects of various parameters, such as, matrix tilt angle, spacers' permeability, wettability and effective contact surface area on the oil production. To provide a better understanding of the reinfiltration process and block-toblock interactions through visualization of the saturation profiles, numerical modelling of free-fall gravity drainage, including block-to-block interaction processes, was performed by developing a CFD model and verified by experimental data obtained in this study.

This research has features, which make it distinct from other studies of this field. First of all, a multi-block experimental set-up is used to quantify the effect of different characteristics of horizontal fracture on the block-to-block interactions. Second, the effect of horizontal fracture wettability was also investigated on matrix block integrity through gas wetting alteration of the utilized spacers. Moreover, COMSOL (Multiphysics 2012) software is used to model the gravity drainage, which was validated against the experimental data. It is shown by visualizing saturation profiles at different times that the reinfiltration process can also be modelled by COMSOL. The presented experimental results can be used in commercial simulators and for validation of numerical models.

In the remainder of the paper first, the numerical simulation backgrounds and governing equations are presented in Sect. 2. Then, experimental materials and method are presented 
in Sect. 3. The experimental and modelling results and discussions are presented in Sect. 4, followed by the summary and concluding remarks.

\section{Numerical Simulation Backgrounds}

Among commercial software packages the multiphysics flexibility of COMSOL (Multiphysics 2012) makes it an appropriate option to study different fluid flow in porous media problems. Bogdanov et al. (2007) investigated steam-assisted gravity drainage mechanism for heavy oil reservoirs using COMSOL. Following that, the process was modelled by STARS package from CMG (Stars and Guide 2008) and the results were compared. They aimed to investigate the adequacy of COMSOL in the modelling of unconventional reservoirs and showed that many important areas of EOR methods can be investigated using this package. Finally, they concluded that, although the computational cost and performance of the commercial packages, like CMG, is still better than COMSOL, due to the high variability and flexibility, COMSOL can be used for phenomenological investigation of new EOR methods in pore to core scale (Bogdanov et al. 2007; Rokhforouz and Amiri 2019). Bjørnarå and Aker (2008) implemented two-phase flow equations of porous media in COMSOL, which can be very complex depending on the introduced assumptions. These equations usually consist two partial differential equations with some auxiliary algebraic equations. The main objective was to determine the appropriate equations for complex modelling (Bjørnarå and Aker 2008). They compared six equations, including partial pressure and fluid flow formulation based on pressure and four other equations were phase pressure-saturation formulation (Binning and Celia 1999), fractional flow (Binning and Celia 1999; Chen et al. 2006), weighted pressure formulation (Chen and Huan 2003; Chen et al. 2006), and Buckley and Leverett (1942) equations based on the pressure and saturation. General form of all mentioned equations is as follows:

$$
\left\{\begin{array}{l}
\sum S_{\alpha}=1, P_{c}=P_{n w}-P_{n}, S_{e \alpha}=f\left(P_{c}\right) \\
\frac{\partial}{\partial t}\left(\phi \rho_{\alpha} S_{\alpha}\right)-\nabla \cdot\left[\rho_{\alpha} \mathbb{K} \frac{k_{r \alpha}}{\mu_{\alpha}}\left(\nabla P_{\alpha}+\gamma_{\alpha} \nabla z\right)\right]=\rho_{\alpha} q_{\alpha} \\
S_{w \alpha}=\frac{S_{w}-S_{w r}}{1-S_{w r}},
\end{array}\right.
$$

where $S_{\alpha}$ and $S_{e \alpha}$ denote the saturation and the effective saturation of the phase $\alpha$, respectively. $P_{c}$ is the capillary pressure, $n w, w$ and $r$ subscripts denote non-wetting, wetting phases and residual saturation, respectively. $\phi$ is porosity, $t$ is time, $\rho$ is density, $\mathbb{K}$ is the medium intrinsic permeability tensor (absolute permeability in case of homogeneous and isotropic assumption), $k_{r}$ is the phase relative permeability, $\mu$ is phase viscosity, $\gamma$ is fluid specific weight, $z$ is the depth and $q$ is the volumetric flow rate. Zendehboudi et al. (2012) modelled free-fall and forced gravity drainage mechanisms using COMSOL, and the simulation results were compared with experimental data. The main objective of this study was to develop a numerical model for gravity drainage in fractured porous media, in addition to describing the effects of various parameters on the process.

\subsection{Governing Equations}

In this study, the matrix was considered as a homogeneous (constant porosity and isotropic permeability) medium. As the pressure gradient was negligible, fluid density was assumed to be constant over time and space (incompressible flow). All similar mathematical models were 
developed based on continuity equation, momentum balance (Darcy law in porous media) and capillary pressure functions. The general form of the governing equations for incompressible multiphase flow (without sink and source terms) for non-wetting and wetting phases, in the SI system can be formulated as:

$$
\begin{gathered}
-\phi \frac{\partial S_{w}}{\partial t}+\nabla \cdot\left(\frac{K k_{r w}}{\mu_{w}}\left(\nabla P_{w}+\rho_{w}\right)\right)=0 \\
-\phi \frac{\partial S_{n w}}{\partial t}+\nabla \cdot\left(\frac{K k_{r n w}}{\mu_{n w}}\left(\nabla P_{n w}+\rho_{n w}\right)\right)=0
\end{gathered}
$$

where $K$ is the absolute permeability and $k_{r}$ is relative permeability. Other used equations for two-phase flow simulation, are capillary pressure and saturation equations, which can be expressed as follows:

$$
\begin{gathered}
P_{c}=P_{n w}-P_{w} \\
S_{n w}+S_{w}=1
\end{gathered}
$$

For implementation of the above governing equations into COMSOL software, there is a need for rearrangement of Eqs. 2-5 (Multiphysics 2012). So, the obtained equations can be expressed as follows:

$$
\begin{gathered}
\phi \frac{\partial S_{w}}{\partial S_{e}} \frac{\partial S_{e}}{\partial P_{c}} \frac{\partial\left(P_{n w}-P_{w}\right)}{\partial t}+\nabla \cdot\left(\frac{K k_{r w}}{\mu_{w}} \nabla P_{w}+\frac{K k_{r w}}{\mu_{w}} \rho_{w} g\right)=0 \\
\phi \frac{\partial S_{w}}{\partial S_{e}} \frac{\partial S_{e}}{\partial P_{c}} \frac{\partial\left(P_{n w}-P_{w}\right)}{\partial t}+\nabla \cdot\left(\frac{K k_{r n w}}{\mu_{n w}} \nabla P_{n w}+\frac{K k_{r n w}}{\mu_{n w}} \rho_{n w} g\right)=0
\end{gathered}
$$

\subsection{Capillary Pressure Function}

To eliminate time derivatives of saturation and rearranging the equation in pressure form, $\frac{\partial S_{e}}{\partial P_{c}}$ was used (Eqs. 6 and 7 ). Capillary pressure is a function of saturation and is used for saturation profile calculation. There are several sets of capillary pressure and relative permeability functions in the literature such as Brooks and Corey (1964), Brutsaert (1966), Mualem (1976) and Van Genuchten (1980). Brooks and Corey (1964) function have been chosen for this study, which can be expressed as follows:

$$
\begin{aligned}
& \begin{cases}S_{e}=\left(\frac{P_{c t}}{P_{c}}\right)^{\lambda}, & P_{c}>P_{c t} \\
=1, & P_{c} \leq P_{c t}\end{cases} \\
& \begin{cases}K_{r w}=S_{e}^{\frac{2+3 \lambda}{\lambda}}, & P_{c}>P_{c t} \\
=1, & P_{c} \leq P_{c t}\end{cases}
\end{aligned}
$$




$$
\begin{cases}K_{r n w}=\left(1-S_{e}\right)^{2}\left(1-S_{e}^{\frac{2+\lambda}{\lambda}}\right), & P_{c}>P_{c t} \\ =1, & P_{c} \leq P_{c t}\end{cases}
$$

where $P_{c t}$ (threshold pressure) and $\lambda$ (pore size distribution index) are characteristic constants of the medium. By defining two separate media in the model (matrix and fracture), with different porosity, permeability and flow function the model geometrical properties were developed. The continuity at the boundary was considered. Capillary pressure value in fractures was assumed nonzero and relative permeability of the fracture was set to be a linear function (Firoozabadi and Ishimoto 1994). Threshold pressure was obtained from Leverett J-function (Leverett 1941), so due to physical property differences, the threshold pressure of the matrix and fracture were different (Sahimi 2011; Zendehboudi et al. 2012; Dejam and Hassanzadeh 2018; Dejam 2018). The numerical model boundary conditions, mesh configuration and validation are further discussed in Sect. 4.2.

\section{Experimental Materials and Methods}

To understand how reinfiltration and capillary continuity mechanisms affect the gravity drainage process, a three-block apparatus was designed and constructed. It was used to speculate the contribution of the block-to-block interactions, which led to higher ultimate recovery factor. The schematic diagram of the fractured packed multi-block model is illustrated in Fig. 1. The set-up has three inner cylinders of $40 \mathrm{~cm}$ height each and the outer cylinder is $120 \mathrm{~cm}$ in height. $0.4 \mathrm{~mm}$ diameter spots on the inner blocks walls were created using a laser cutter, which connects the matrix blocks to the vertical fracture. The number and diameter of the spots were controlled by the existence of hydraulic continuity between the two media. According to the provided explanation, 400 spots per $40 \mathrm{~cm}$ of the model was considered and uniformly performed. Two horizontal fractures were designed between the blocks. The use of spacers represents these horizontal fractures, and as the properties of the spacers changed, the significance of the block-toblock interaction mechanism could be modified accordingly. The utilized spacers and their characteristics are presented in Table 1. The annulus between inner cylinders and the outer cylinder represented the vertical fracture. The flow was controlled by two inlet and outlet valves and the outlet stream accumulated in a glass container, which was closed with a cap to avoid fluid evaporation. Weight of the produced fluid was measured using an electronic balance and the production data were logged by a data acquisition system.

Free-fall gravity drainage experiments were conducted at ambient condition $\left(20{ }^{\circ} \mathrm{C}, 1\right.$ atm). During the experiment, the top of the model was open to the atmosphere by opening the inlet valve. The details of the experimental procedure, including sand packing, fluid saturation and data acquisition are as follows:

1. Specific amount of sand (with a size range of $0.2-0.4 \mathrm{~mm}$ ) was weighed and placed inside of each inner cylinder as the matrix block. The cylinder was placed on a shaker to obtain a uniform and homogeneous sand pack. The process continued until no more sand could be added. 
Table 1 Spacers used for the three-block experiments and their specifications

\begin{tabular}{|c|c|c|c|c|}
\hline Type & $\begin{array}{c}\text { Permeability } \\
(\mathbf{m d})\end{array}$ & $\begin{array}{c}\text { Thickness } \\
(\mathbf{m m})\end{array}$ & Material & Representation \\
\hline Full contact & - & Less than 0.1 & Steel mesh & \\
\hline Barrier & - & 1 & $\begin{array}{c}\text { Aluminium } \\
\text { sheet }\end{array}$ & \\
\hline $\begin{array}{c}\text { Thin section } \\
\text { (type I) }\end{array}$ & 100 & 0.5 & $\begin{array}{c}\text { High } \\
\text { permeability } \\
\text { thin section }\end{array}$ & \\
\hline $\begin{array}{c}\text { Thin section } \\
\text { (type II) }\end{array}$ & 20 & 0.5 & $\begin{array}{c}\text { Low } \\
\text { permeability } \\
\text { thin section }\end{array}$ & \\
\hline & - & 1 & $\begin{array}{c}\text { Perforated } \\
\text { aluminium sheet }\end{array}$ \\
\hline
\end{tabular}

Fig. 1 Schematic of the stack of three-block experimental set-up

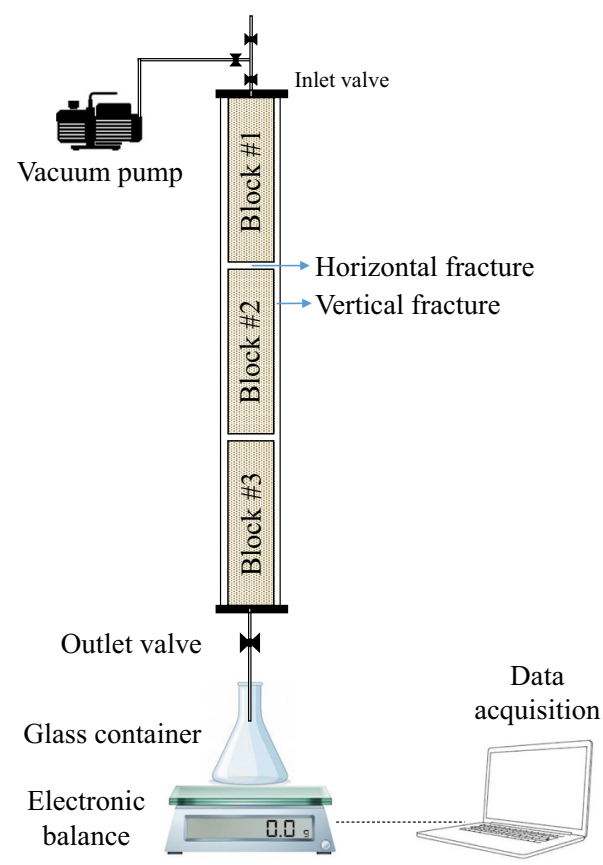

2. The top and the bottom of each inner cylinder were blocked by 400 mesh metal screens. Then, they were placed in the outer cylinder. Afterwards, the upper and lower caps were tightened.

3. The bottom valve was closed and the upper one was opened to the vacuum pump. 
Table 2 Physical specifications of the three-block experimental set-up

Table 3 Test fluids specifications

\begin{tabular}{ll}
\hline Inner cylinders length & $40 \mathrm{~cm}$ \\
Outer cylinder length & $120 \mathrm{~cm}$ \\
Inner cylinders diameter & $2.9 \mathrm{~cm}$ \\
Outer cylinder diameter & $3 \mathrm{~cm}$ \\
Vertical fracture aperture & $0.05 \mathrm{~cm}$ \\
\hline
\end{tabular}

\begin{tabular}{llll}
\hline & Test fluid & Viscosity $(\mathrm{cP})$ & Density $\left(\mathrm{kg} / \mathrm{m}^{3}\right)$ \\
\hline Gas phase & Air & 0.018 & 1.2 \\
Liquid phase & Kerosene & 1.64 & 790 \\
\hline
\end{tabular}

4. A glass container of a specific amount of test fluid was placed on balance and the vacuumed system of matrix and fracture begins to saturate using a line, which connected the bottom valve and test fluid in the container. Subtracting the weight of the beaker after and before the saturation process yielded the amount of imbibed fluid throughout the saturation process. having the bulk volume of the system and the void volume using the imbibed fluid, we were able to calculate the porosity of the sand pack (porous media).

5. The experiment commenced when the upper valve was opened to the atmosphere and the bottom one to the glass container. An electronic balance was used to measure the weight of the produced fluid, which was connected to the data acquisition system, used for logging the data. Moreover, the glass container was closed using parafilm to avoid fluid evaporation during the experiment.

The weight of the container was measured continuously, and the recovery factor was calculated from the indirect volume measurements. It should be noted that using crude oil as the test fluid was restricted due to Plexiglas properties. The inner cylinders were representative of the matrix blocks, and specifications of the multi-block model and the test fluids are presented in Tables 2 and 3, respectively. To investigate the role of horizontal fracture surface wetness, four spacers (thin section type I) were coated by MariSeal 800 chemical as a gas wetting agent (Erfani Gahrooei and Ghazanfari 2017, 2018; Azadi Tabar and Ghazanfari 2019).

\section{Results and Discussion}

\subsection{Experimental Results}

\subsubsection{Effect of Tilt Angle}

To examine the effect of tilt angle on the gravity drainage process in the presence of blockto-block interactions, three angles of $15^{\circ}, 6^{\circ}$, as well as $0^{\circ}$ from the vertical direction, were selected for the experiments. Moreover, to activate the reinfiltration mechanism, direct contact spacers were placed between the blocks. Figure 2 presents the obtained experimental data for different tilt angles, which shows that increasing the tilt angle of the system leads 
Fig. 2 Effect of tilt angle on recovery factor in the three-block system

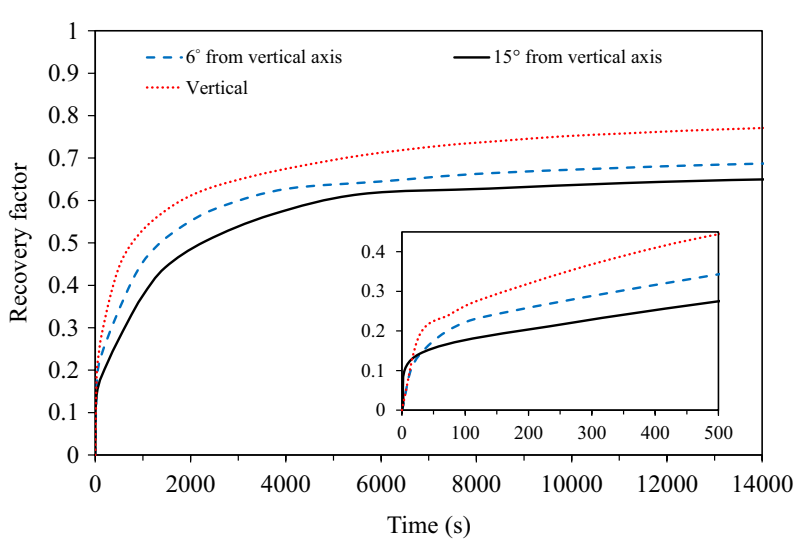

to a higher ultimate recovery factor. Increasing the tilt angle decreases the effective height of the system so weakens the gravity force. Moreover, in higher tilt angles the travelling liquid blocks will be transferred easier to the vertical fracture rather than forming liquid bridges in the horizontal fracture. So, increasing the tilt angle can decrease the capillary continuity. Noteworthy, the obtained ultimate recovery factors were in the same range of the obtained experimental data by other researchers (Firoozabadi and Markeset 1992, 1994), while quantitative comparison was avoided because of different set-up configuration (such as block height and annulus thickness), rock and fluid properties. The capillary gravity ratio (CGR) for the gravity drainage process can be defined as

$$
C G R=\frac{\Delta \rho g H}{2 \sigma \cos (\theta) / r}
$$

where $H$ is the block height, $\sigma$ is kerosene-air surface tension ( 30 dyne/cm), $\theta$ is kerosenerock-air contact angle and $r$ is the average pore size. The CGR was calculated as $\approx 10$ for the experimental condition, which shows the dominance of gravity force over capillary in our experiments. In higher tilt angles the effective block height $(\mathrm{H})$ and the CGR decrease, which results in lower recovery factors.

Mentioned mechanisms are the most significant fluid transfer mechanisms from one block to another. Moreover, the effective height of the system is decreased by the increase of the tilt angle from the vertical plane. In higher tilt angles, the velocity of the travelling droplets decreases and, as a result, the reinfiltration process will be weakened. Thus, in higher tilt angles it is easier for droplets to enter the vertical fracture rather than being imbibed into the lower block, so the liquid bridges become weaker.

\subsubsection{Effect of Spacers' Permeability}

The oil recovery process from a block in a fractured reservoir can be divided into two regimes. The period before gravity-capillary equilibrium (i.e., early time, transient) and the period after these forces balance (i.e., late time, steady state). In early time, blockto-block interactions result in a lower gravity drainage rate, due to reinfiltration and the formation of liquid bridges (i.e., capillary continuity) mechanisms. Interaction between blocks increases the effective height and the ultimate recovery factor. The experiments showed that the upper block did not have a significant role in cumulative recovery at 
the first steps of the process, and it can even postpone the drainage of the lower block. However, by draining the oil from the upper block to the lower, the cumulative recovery of the system is increased. In other words, the drainage of a multi-block system can take more time rather than a single block. Hence, one of the crucial factors in any gravity drainage process is determining the effective height of the blocks in the system.

Hence, it can be concluded that the formation of liquid bridges leads to higher gravity drainage efficiency in late time. It is founded that this mechanism will enhance the drainage rate until the gravity-capillary equilibrium takes place. Overproduction of a multi-blocks system is related to the liquid film phenomenon, which is obtained by direct contact of the blocks, which is controlled by the capillary number and the fracture permeability. Dejam and Hassanzadeh (2011) investigated the critical length of a droplet, which affected the formation of liquid bridges. They showed that the critical length $\left(L_{c}\right)$ depends on a threshold bond number $\left(B_{0}^{*}=\frac{\rho g r^{2}}{\sigma}\right)$ and fracture capillary pressure $\left(P_{c f}\right)$. The relation between these parameters is expressed as follows:

$$
L_{c}=\frac{\sigma}{P_{c f}}\left(\frac{1}{B_{0}^{*}}-2\right)
$$

According to Eq. 12, increasing the fracture capillary pressure leads to a decrease in the critical length. To evaluate the effect of capillary pressure which can be related to the pore size distribution and fracture permeability, spacers with different permeabilities were used in the experiments. Figure 3 depicts the effect of spacers' permeability on the oil recovery factor, in the early time stage the gravity force was dominating the fluid flow. Reducing the fracture permeability, as expressed in Eq. 12 leads to greater critical length and will increase the number of liquid bridges (Dejam and Hassanzadeh 2011, 2018). This phenomenon temporarily decreases the oil production rate. The oil droplets expelled from the upper block will be imbibed into the lower block through liquid bridges. However, in the late time, recovery would increase because of the reduction in threshold height for spacers with lower permeability.

Fig. 3 Effect of spacer permeability on the recovery factor in the three-block system

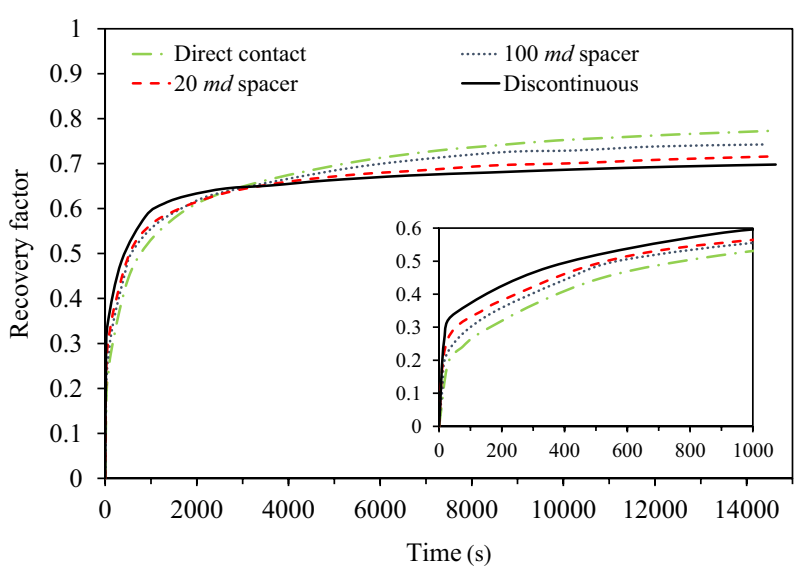




\subsubsection{Effect of Spacers' Wettability}

Spacers' wettability can be considered as a parameter, controlling the horizontal fracture capillary pressure. Equation 13 determines the fracture capillary pressure (Kralchevsky and Nagayama 2001; Ghezzehei and Or 2005) as:

$$
P_{c f} \approx \sigma\left(\frac{1}{r_{0}}-\frac{2 \cos \theta}{b_{f}}\right)
$$

where $r_{0}$ is droplet radius and $\theta$ is the contact angle, which represents the wettability of the fracture, $b_{f}$ is the fracture aperture and $\sigma$ is the interfacial tension. Figure 4 shows the schematic representation of a liquid droplet in a horizontal fracture. According to Eq. 13, which applies to an open fracture, the contact angle of the wetting phase, would be between $0^{\circ}$ and $90^{\circ}$. Therefore, the value of the capillary pressure is less than the condition in which $\theta$ is between $90^{\circ}$ and $180^{\circ}$. As a result, an oil-wet matrix may result in a stronger capillary continuity and a stable liquid bridge formation, which ultimately results in higher oil recovery. Yet, the equation for the fracture capillary pressure is not applicable in this research, because the utilized spacers were porous (thin sections) and capillary pressure model for this condition can be expressed as:

$$
P_{c f} \approx \sigma_{f}\left(\frac{2 \cos \theta}{b_{f}}\right)
$$

Figure 5 illustrates the experimental results of the recovery factor versus time for oilwet and gas-wet spacers. In each experiment, four thin sections of type (I) were placed on the upper and lower faces of the horizontal fractures (direct contact spacer was used as the horizontal fracture). As depicted in Fig. 5 in early time stage lower recovery was obtained in the presence of oil-wet spacer that may occur due to lower fracture capillary pressure and the corresponding block-to-block interactions, but the ultimate recovery factor was higher in this case; which could be explained as a result of better communication between blocks through liquid bridges between the matrix blocks, and subsequent decrease of the threshold height of the system. Additionally, in the gas wetting condition of the horizontal fractures, there is a higher chance of communication with the vertical fracture for the produced oil from a matrix block as the liquid droplets are not absorbed by the fracture walls, so the chance of channelling to the vertical fracture is increased.

Fig. 4 Schematic representation of a liquid droplet in a horizontal fracture (Dejam and Hassanzadeh 2011)

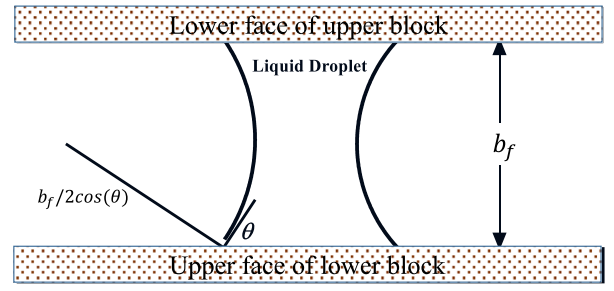


Fig. 5 Effect of spacers' wettability on the recovery factor in the three-block system

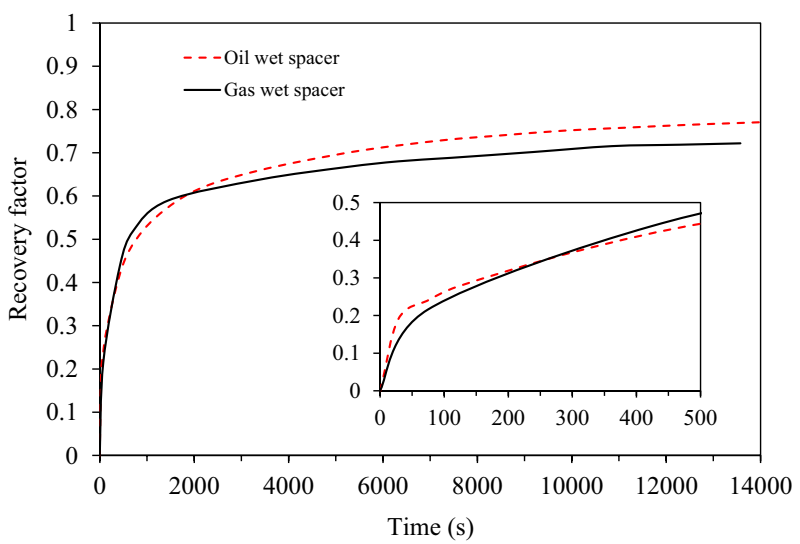

\subsubsection{Effect of Spacers' Effective Surface Area}

Figure 6 presents the obtained experimental results obtained by sparse and direct contact spacers. As shown, direct contact spacers obtained higher ultimate recovery for the system compared to sparse spacers (for the spacers specifications refer to 1). One of the important factors, controlling the reinfiltration phenomena, is the spacers' effective surface area, providing contact between the upper and the lower matrix blocks. As this factor have a direct influence on capillary pressure, reducing the fracture effective area will hypothetically weaken the capillary continuity. Because in such condition, there is less room for the formed oil droplet to increase in size. Besides, heterogeneity and roughness of the fracture surface hinder the film flow and the travelling droplet mechanism. So, greater contact between the blocks results in higher production.

Fig. 6 Effect of spacers' effective surface area on the recovery factor in the three-block system

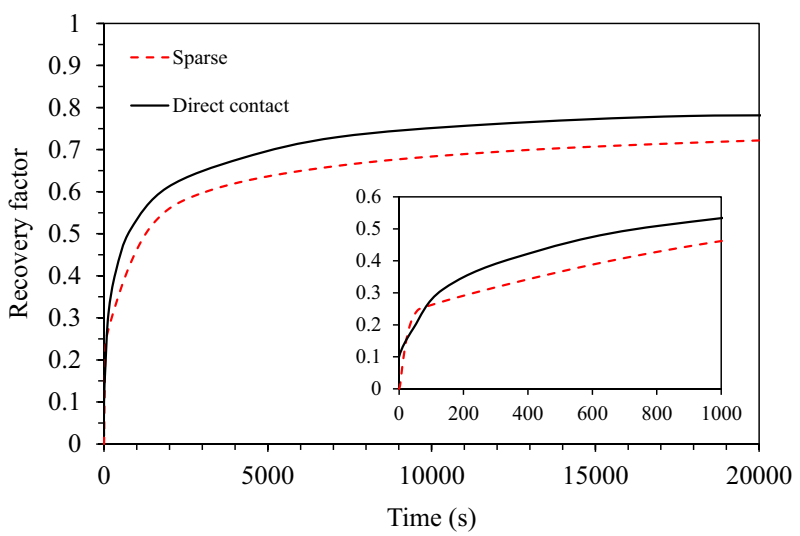


Fig. 7 Schematic view of the boundaries for multi-stack blocks numerical model

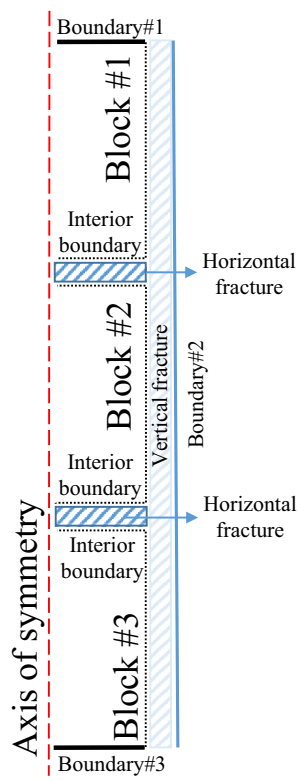

Table 4 Numerical model boundary and initial conditions

\begin{tabular}{lll}
\hline Boundary/initial condition & Oil & Gas \\
\hline Initial condition & $P_{w}(z)=\left(-\rho_{w} \times g H-P_{d f}\right) \times \frac{z}{H}$ & $P_{n w}(z)=\rho_{n w} g z$ \\
Interior boundary & Continuity & Continuity \\
Boundary\#1 & - & $P_{n w}(z)=\rho_{n w} g H$ \\
Boundary\#2 & No flow & No flow \\
Boundary\#3 & $P_{w}=0$ & - \\
\hline
\end{tabular}

\subsection{Numerical Simulation Results}

\subsubsection{Model Geometry and Boundary Conditions}

Figure 7 illustrates the schematic view of the boundaries for multi-stack blocks numerical model. At initial condition, the model was saturated with oil and boundary\#1 was opened into the air and atmospheric pressure and boundary\#3 had atmospheric pressure as well. Boundary\#2 was non-wet for both phases and no-flow boundary condition was considered at this face. Boundary and initial conditions for the model are summarized in Table 4.

\subsubsection{Mesh Configuration}

For a model with a small grid size, the software will provide more accurate results, but the runtime increases for each simulation. Since fluid mostly flows in the vertical direction, a rectangular grid leads to acceptable results compared to triangular mesh. Using the finite 
Table 5 Multi-block COMSOL model specifications

\begin{tabular}{lll}
\hline Parameter & Value at fracture & Value at matrix block \\
\hline$K\left(\mathrm{~m}^{2}\right)$ & $2 \times 10^{-8}$ & $6 \times 10^{-10}$ \\
$P_{d}(\mathrm{~Pa})$ & 100 & 500 \\
$\lambda$ & 4 & 2 \\
$S_{w r}$ & - & 0.25 \\
$\phi$ & 1 & 0.4 \\
$\rho_{o}\left(\mathrm{~kg} / \mathrm{m}^{3}\right)$ & 790 & 790 \\
$\rho_{g}\left(\mathrm{~kg} / \mathrm{m}^{3}\right)$ & 1.2 & 1.2 \\
$\mu_{o}(\mathrm{~Pa} \mathrm{~s})$ & $1.6 \times 10^{-3}$ & $1.6 \times 10^{-3}$ \\
$\mu_{g}(\mathrm{~Pa} \mathrm{~s})$ & $1.8 \times 10^{-5}$ & $1.8 \times 10^{-5}$ \\
\hline
\end{tabular}

Fig. 8 Validation of the threeblock numerical model with the experimental data

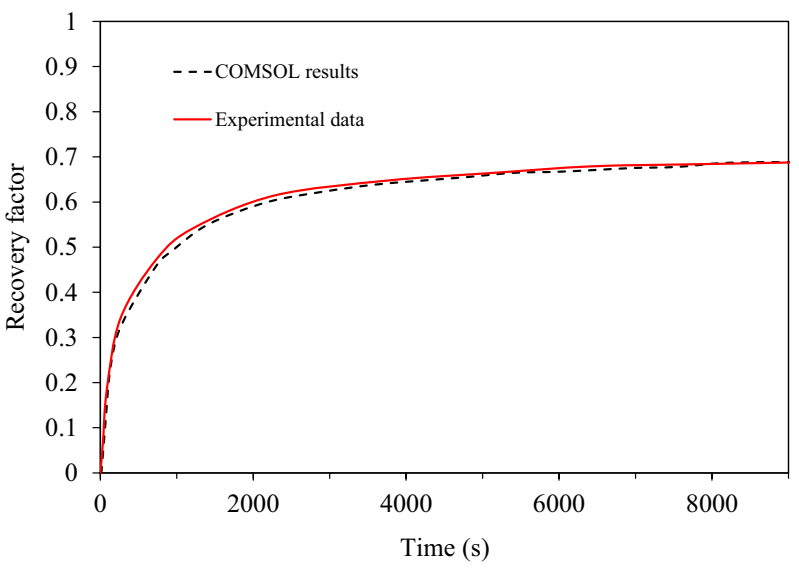

element method, which can simply handle the front displacement, fine-grid mapping may not seem to be necessary due to low gradient fluid flow (i.e., free-fall gravity drainage). To ensure the accuracy of the analysis, the effect of grid size on the obtained results was investigated by varying the mesh size to find the optimal size for further calculations.

\subsubsection{Model Validation}

In this section, model validation against the experimental results, and estimation of the Corey model saturation function parameters are presented. As the model parameters were estimated based on the experimental results and the characteristics of the conducted experiments, some model properties especially the fracture and matrix permeability are different from typical values in the field. Table 5 presents the obtained model parameters.

Figure 8 shows an acceptable match between the experimental data and the numerical model results. The experiment was conducted with sea packed sand and kerosene using direct contact spacers. The horizontal fracture aperture in the numerical model was $0.01 \mathrm{~mm}$ and the matrix permeability was 600 Darcy. Table 6 compares the recovery factor results for the experiment and the numerical model for three different times. The presented data illustrate the 
Table 6 Results of experiment and numerical modelling at different times

\begin{tabular}{lll}
\hline Time (s) & Recovery factor (experiment) & $\begin{array}{l}\text { Recovery } \\
\text { factor } \\
\text { (model) }\end{array}$ \\
\hline 600 & 0.48 & 0.47 \\
2400 & 0.62 & 0.60 \\
10,000 & 0.66 & 0.66 \\
\hline
\end{tabular}

fact that the numerical modelling results are in good agreement with the experimental data. The average relative error was less than $5 \%$ throughout the simulation compared to the experiment, which is acceptable.

\subsubsection{Effect of Mesh Size on the Numerical Solution}

Figure 9 presents the numerical modelling results for three different element numbers; the presented results prove that the obtained results are independent of both mesh size and mesh number, in the selected range. So, the coarse mesh (150 elements) was selected for further calculations as it had lower runtime. The mesh was refined at horizontal fractures and we made sure to use at least 5 mesh elements in the fracture aperture.

\subsubsection{Application of COMSOL for Simulating the Reinfiltration Phenomena}

Figures 10 and 11 illustrate the oil saturation profiles along the three-block system used in the experiments with uniform block permeability and horizontal fracture aperture of $0.1 \mathrm{~mm}$ obtained from the numerical model, at early and late times, respectively. Before any discussion, it may be helpful to briefly review the concepts of reinfiltration phenomenon. Equation 15 presents one dimensional drainage rate at the depth of $z=0$ (lower surface) and reinfiltration at $z=H$ (upper surface):

$$
q=\frac{K k_{r o}}{\mu_{o}}\left[\Delta \rho g-\frac{\partial P_{c}}{\partial S_{o}} \frac{\partial S_{o}}{\partial z}\right]
$$

Fig. 9 Effect of mesh size and number on the numerical results

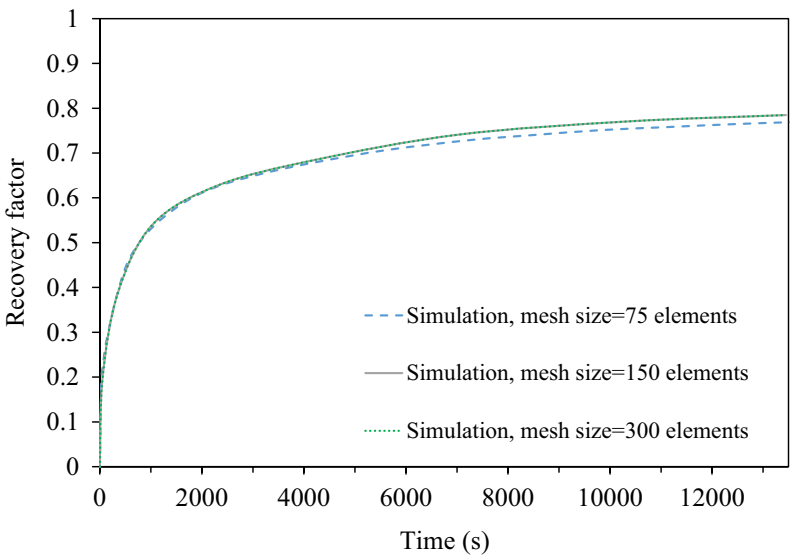


Fig. 10 Early time simulation results of oil saturation profiles for the three-block system. (Note: the horizontal axis shows the model elevation and the oil saturation is shown in the vertical axis)

Fig. 11 Late time simulation results of oil saturation profiles for the three-block system. (Note: the horizontal axis shows the model elevation and the oil saturation is shown in the vertical axis)
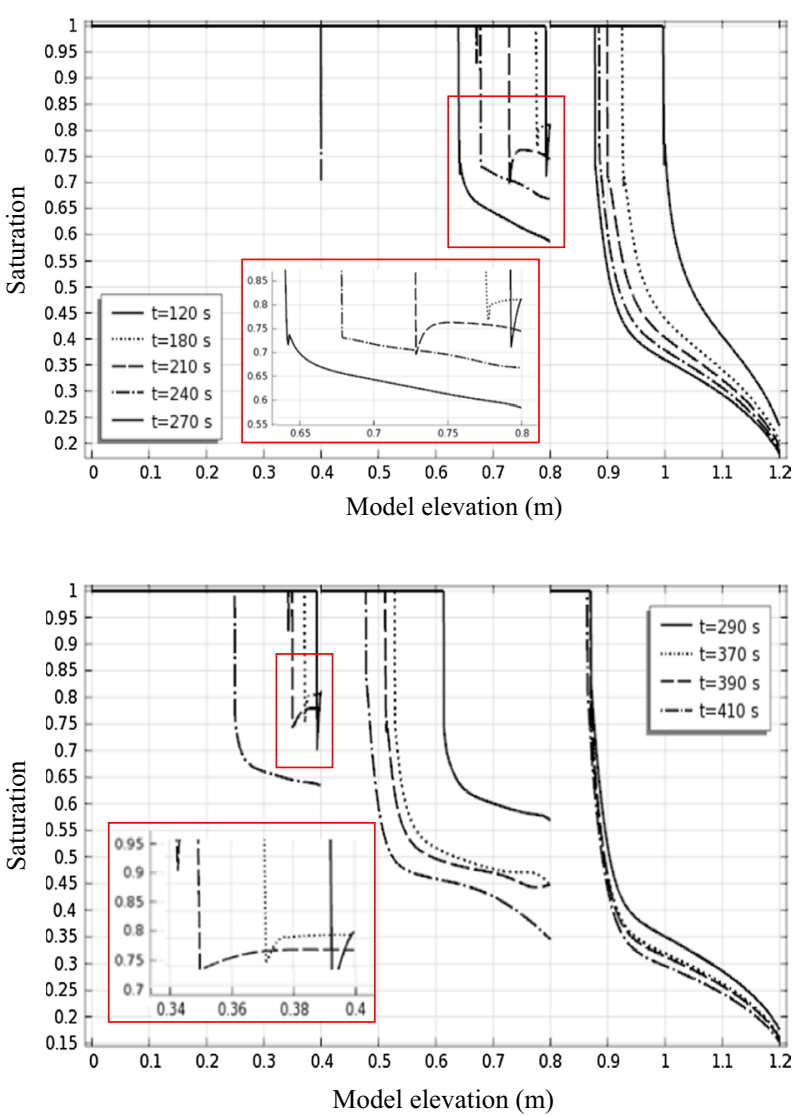

The term $\frac{\partial S_{o}}{\partial z}$ can be positive, negative or zero. It can be negative when the threshold pressure is zero. At $z=0$, where the block is fully saturated with oil, $\frac{\partial S_{o}}{\partial z}$ is zero. If the threshold pressure is zero, the maximum rate of production at $z=0$ which is the initial rate reads as:

$$
q=\frac{K k_{r o}}{\mu_{o}}[\Delta \rho g]
$$

When the production from the block at $z=0$ begins, $\frac{\partial S_{o}}{\partial z}$ is negative and the rate of drainage decreases, so the rate of drainage at $z=0$ follows:

$$
q \leqslant \frac{K k_{r o}}{\mu_{o}}[\Delta \rho g]
$$

If reinfiltration exists at the top of the block, a sufficient amount of fluid can be fed from the upper boundary and $S_{o}$ at $z=0$ equals to 1 , as the saturation gradient at $z=H$ is zero, while if an insufficient amount of fluid was fed from above, $S_{o}$ at $z=0$ equals to 1 and the saturation gradient at $z=H$ will be positive, so: 


$$
q \geqslant \frac{K k_{r o}}{\mu_{o}}[\Delta \rho g]
$$

As it is shown in Fig. 10 the saturation profiles indicate a change or return at $120 \mathrm{~s}$ time at the location of the horizontal fracture. The reason for this sudden increase in oil saturation at the horizontal fracture is the emergence of inter-block phenomena such as the formation of liquid bridges and reinfiltration of the produced oil from the upper block and it can increase the oil saturation in the lower block because of the imbibed oil into this block. Since pressure continuity boundary condition was applied at the boundary of the horizontal fractures and the adjacent blocks, the simulator was able to model inter-block phenomena, causing such sudden change in properties (e.g,. permeability, porosity and saturation functions) of two media (matrix block and fracture). In other words, with mesh refinement at the boundary of the fracture and the use of the finite element method, we could study the effect of the block-to-block interactions on the gravity drainage process efficiency. At times of 180 and $210 \mathrm{~s}$ feeding the lower block from the upper one through inter-blocks phenomena is obvious from the saturation profile. For instance, oil saturation at $210 \mathrm{~s}$ for approximate heights of 0.73 to 0.8 is equal despite oil production. This represents compensation of the oil production with the oil fed from the upper block, being imbibed to the lower one. At times 240 and $270 \mathrm{~s}$ the upper block was completely drained and is at residual oil saturation, so the saturation profile of the middle block followed a normal distribution.

It can be concluded that the oil saturation of the upper block should be less than the lower block in both gravity drainage and reinfiltration process; however, these phenomena are more obvious in reinfiltration process because of the imbibed oil into the underneath block.

\subsubsection{Effect of Horizontal Fracture Aperture}

In this section, we study the effect of horizontal fracture aperture on the gravity drainage performance, which was experimentally difficult to study. As Fig. 12 shows, decrease in the horizontal fracture aperture stimulates the block-to-block interactions such as reinfiltration, film flow, travelling liquid droplets and capillary continuity through the formation of the liquid bridge; thus, the rate of oil production in early times decreases due to presence of such mechanisms. However, due to capillary continuity, the threshold

Fig. 12 Effect of horizontal fracture aperture on the recovery factor of the multi-stack block system. (All fracture aperture units are in $\mathrm{mm}$ )

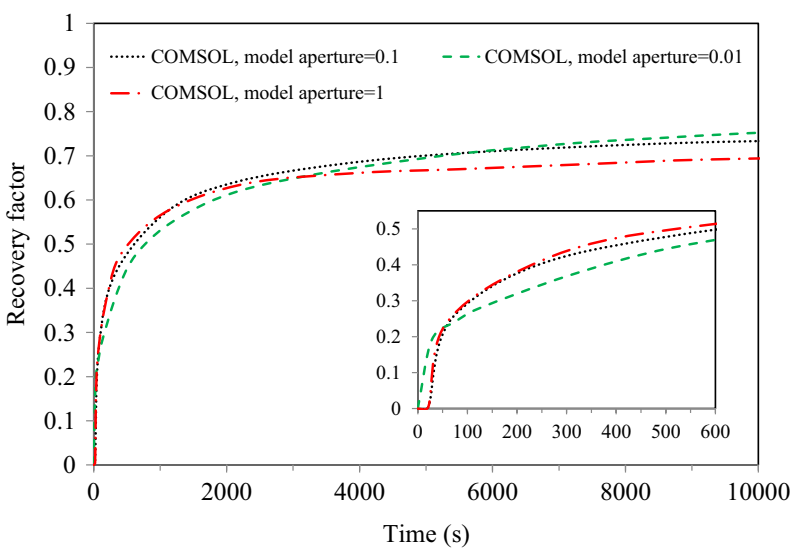


height decreases and the ultimate recovery is increased. Moreover, when the fracture aperture increases, the capillary pressure in the fracture is decreased which leads to unstable liquid bridges (refer to Eqs. 12-14). Such phenomena decrease the oil drainage rate in early times. Another reason behind increasing the ultimate recovery in late time is lower liquid saturation in the horizontal fractures. In conclusion, stable liquid bridges, which occur in smaller fracture apertures, can maintain the capillary continuity and increase the ultimate recovery factor of the gravity drainage process.

\section{Summary and Conclusions}

In this work, an experimental set-up, as well as a numerical model of a multi-blocks system, was successfully designed and used to analyse the gravity drainage mechanism in fractured systems. The effects of capillary continuity and reinfiltration phenomena, which mainly control block-to-block interactions, were investigated. The impacts of important parameters such as tilt angle, spacers' wettability, permeability, effective surface were investigated experimentally using an intensive experimental set. COMSOL multiphysics package was used for numerical modelling of the process to understand the block-to-block interactions through visualization of the saturation profiles. The numerical model was validated by the experimental data. Based on the experimental data and modelling results presented in this work, the following conclusions can be drawn:

- Decreasing the horizontal fractures aperture decreases oil drainage rate in early time stages but increases the ultimate recovery due to the greater extent of capillary continuity between the adjacent blocks.

- Tilt angle from the vertical axis decreases the recovery factor, due to higher velocity of travelling liquid element and liquid film flow as well as a higher chance for oil droplets to enter the vertical fracture rather than being imbibed by the underneath matrix block. Moreover, increasing the tilt angle of the system decreases the effective height of the system and lowers the gravity compared to capillary force and hence decreases the ultimate recovery factor.

- Gas wetting alteration of the horizontal fractures weakens the capillary continuity and decreases the ultimate recovery factor, compared to original liquid wetting condition. Conversely, the model with gas-wet horizontal fractures showed a higher oil production rate in early time stages, due to better communication of the matrix blocks with the vertical fracture.

- Reinfiltration contribution in the block-to-block interaction process is mainly controlled by the horizontal fracture specifications. Higher spacers' permeability decreases the oil production rate at early times, while it increases the ultimate recovery factor due to higher capillary continuity and lower system threshold height.

- Good agreement was observed between the numerical model results and the experimental data of oil recovery, showing that COMSOL multiphysics can successfully be used for the modelling of the gravity drainage process in a multi-block system. The validated numerical model was used to visualize the saturation profiles and study the effect of horizontal fracture aperture on the gravity drainage process in a multi-block system. 
- The behaviour of the saturation profiles along with the matrix blocks, obtained from the numerical modelling, showed that block-to-block interaction phenomenon was successfully modelled by COMSOL CFD software.

The provided experimental data and modelling results shed light into the effect of different fracture properties on the block-to-block interactions and their interplay on the ultimate recovery factor of the system. Yet there are many other important factors which can be further studied by designing specific experimental set-ups or numerical models. Some of these factors include the effect of production rate on block-to-block interactions, the heterogeneity of the matrix blocks and the effect of heterogeneity sequence and layering, fracture roughness and the vertical fracture properties.

Acknowledgements The authors would like to acknowledge Mr. FKM for his great and invaluable help with designing and construction of the experimental set-up as well as conducting the experiments. We are also thankful to Mr. Mohammad Javad Shokriafra, Mr. Hamidreza Norouzi and Mr. Haj Alireza Ghaderi Ardakani for their scientific support and constructive discussions.

Data Availability Statement The experimental data presented in this research are available upon request from the corresponding author for research/non-commercial purposes.

\section{Compliance with Ethical Standards}

Conflict of interest The authors declare no competing financial interest.

Open Access This article is licensed under a Creative Commons Attribution 4.0 International License, which permits use, sharing, adaptation, distribution and reproduction in any medium or format, as long as you give appropriate credit to the original author(s) and the source, provide a link to the Creative Commons licence, and indicate if changes were made. The images or other third party material in this article are included in the article's Creative Commons licence, unless indicated otherwise in a credit line to the material. If material is not included in the article's Creative Commons licence and your intended use is not permitted by statutory regulation or exceeds the permitted use, you will need to obtain permission directly from the copyright holder. To view a copy of this licence, visit http://creativecommons.org/licenses/by/4.0/.

\section{References}

Abbasi, M., Rostami, P., Moraveji, M.K., Sharifi, M.: Generalized analytical solution for gravity drainage phenomena in finite matrix block with arbitrary time dependent inlet boundary condition and variable matrix block size. J. Petrol. Sci. Eng. 167, 227-240 (2018). ISSN 0920-4105

Aghabarari, A., Ghaedi, M.: A new simulation approach to investigate reinfiltration phenomenon in fractured porous media. J. Petrol. Sci. Eng. (2019). ISSN 0920-4105

Akhlaghi, N., Kharrat, R., Mahdavi, S.: Gas assisted gravity drainage by $\mathrm{CO}_{2}$ injection. Energy Sources Part A Recov. Util. Environ. Eff. 34(17), 1619-1627 (2012)

Aljuboori, F.A., Lee, J.H., Elraies, K.A., Stephen, K.D.: Gravity drainage mechanism in naturally fractured carbonate reservoirs; review and application. Energies 12(19), 3699 (2019)

Ameri, A., Farajzadeh, R., Suicmez, V., Verlaan, M., Bruining, J.: Dynamic interactions between matrix and fracture during miscible gravity drainage in naturally fractured reservoirs. Ind. Eng. Chem. Res. 54(19), 5356-5371 (2015). ISSN 0888-5885

Aminnaji, M., Rabbani, A., Niasar, V.J., Babaei, M.: Effects of pore-scale heterogeneity on macroscopic NAPL dissolution efficiency: a two-scale numerical simulation study. Water Resour. Res. 55(11), 8779-8799 (2019)

An, S., Erfani, H., Godinez Brizuela, O.E., Niasar, V.: Transition from viscous fingering to capillary fingering: application of gpu-based fully-implicit dynamic pore-network modelling. Water Resour. Res. e2020WR028149 (2020a) 
An, S., Erfani, H., Hellevang, H., Niasar, V.: Lattice-Boltzmann simulation of dissolution of carbonate rock during $\mathrm{CO}_{2}$-saturated brine injection. Chem. Eng. J. 127235 (2020b)

An, S., Hasan, S., Erfani, H., Babaei, M., Niasar, V.: Unravelling effects of the pore-size correlation length on the two-phase flow and solute transport properties; GPU-based pore-network modelling. Water Resour. Res. e2020WR027403 (2020c)

Austad, T., Standnes, D.C.: Spontaneous imbibition of water into oil-wet carbonates. J. Petrol. Sci. Eng. 39(3-4), 363-376 (2003)

Azadi Tabar, M., Ghazanfari, M.: Experimental study of surfapore nanofluid effect on surface free energy of calcite rock. Modares Mech. Eng. 19(3), 709-718 (2019)

Aziz, R., Niasar, V., Erfani, H., Martínez-Ferrer, P.J.: Impact of pore morphology on two-phase flow dynamics under wettability alteration. Fuel 268, 117315 (2020)

Babaei, M., Islam, A.: Convective-reactive co2 dissolution in aquifers with mass transfer with immobile water. Water Resour. Res. 54(11), 9585-9604 (2018)

Babaei, M., Nick, H.M.: Performance of low-enthalpy geothermal systems: interplay of spatially correlated heterogeneity and well-doublet spacings. Appl. Energy 253, 113569 (2019)

Bahari Moghaddam, M., Rasaei, M.R.: Experimental study of the fracture and matrix effects on free-fall gravity drainage with micromodels. SPE J. 20(02), 324-336 (2015)

Bakhshi, P., Kharrat, R., Hashemi, A., Zallaghi, M.: Experimental evaluation of carbonated waterflooding: a practical process for enhanced oil recovery and geological $\mathrm{CO}_{2}$ storage. Greenh. Gases Sci. Technol. 8(2), 238-256 (2018)

Bech, N., Jensen, O.K., Nielsen, B.: Modeling of gravity-imbibition and gravity-drainage processes: analytic and numerical solutions. SPE Reserv. Eng. 6(01), 129-136 (1991). ISSN 0885-9248

Binning, P., Celia, M.A.: Practical implementation of the fractional flow approach to multi-phase flow simulation. Adv. Water Resour. 22(5), 461-478 (1999)

Bjørnarå, T.I., Aker, E.: Comparing equations for two-phase fluid flow in porous media. In: COMSOL conference, Hannover (2008)

Bogdanov, I., El Ganaoui, K., Kamp, A.: COMSOL 2D simulation of heavy oil recovery by steam assisted gravity drainage. In: Proceedings of the European COMSOL Conference (2007)

Brooks, R.H., Corey, A.T.: Hydraulic properties of porous media and their relation to drainage design. Trans. ASAE 7(1), 26-28 (1964)

Brutsaert, W.: Probability laws for pore-size distributions. Soil Sci. 101(2), 85-92 (1966). ISSN 0038-075X

Buckley, S., Leverett, M.: Mechanism of fluid displacement in sands. Trans. AIME 146(01), 107-116 (1942)

Chatzis, I., Ayatollahi, S.: The effect of gas injection rate on the recovery of waterflood residual oil under gravity assisted inert gas injection. In: Technical Meeting/Petroleum Conference of The South Saskatchewan Section. Petroleum Society of Canada (1993)

Chatzis, I., Kantzas, A., Dullien, F.: On the investigation of gravity-assisted inert gas injection using micromodels, long Berea sandstone cores, and computer-assisted tomography. In: SPE Annual Technical Conference and Exhibition. Society of Petroleum Engineers (1988)

Chen, Y., Ubaidah, A., Elakneswaran, Y., Niasar, V.J., Xie, Q.: Detecting ph and $\mathrm{Ca}^{2+}$ increase during low salinity waterflooding in carbonate reservoirs: implications for wettability alteration process. J. Mol. Liq. 114003 (2020)

Chen, Z., Huan, G.: Numerical experiments with various formulations for two phase flow in petroleum reservoirs. Transp. Porous Media 51(1), 89-102 (2003)

Chen, Z., Huan, G., Ma, Y.: Computational Methods for Multiphase Flows in Porous Media. SIAM, Philadelphia (2006)

Chow, L., Butler, R.: Numerical simulation of the steam-assisted gravity drainage process (SAGD). J. Can. Petrol. Technol. 35(06), 90-96 (1996)

Dejam, M.: The role of fracture capillary pressure on the block-to-block interaction process. J. Porous Media 21(11), 1121-1136 (2018)

Dejam, M.: Advective-diffusive-reactive solute transport due to non-newtonian fluid flows in a fracture surrounded by a tight porous medium. Int. J. Heat Mass Transf. 128, 1307-1321 (2019)

Dejam, M., Hassanzadeh, H.: Formation of liquid bridges between porous matrix blocks. AIChE J. 57(2), 286-298 (2011). ISSN 1547-5905

Dejam, M., Hassanzadeh, H.: The role of natural fractures of finite double-porosity aquifers on diffusive leakage of brine during geological storage of co2. Int. J. Greenh. Gas Control 78, 177-197 (2018)

Dejam, M., Ghazanfari, M.H., Kamyab, M., Masihi, M.: The gas-oil gravity drainage model in a single matrix block: a new relationship between relative permeability and capillary pressure functions. J. Porous Media 14(8), 709-720 (2011)

Di Donato, G., Tavassoli, Z., Blunt, M.J.: Analytical and numerical analysis of oil recovery by gravity drainage. J. Petrol. Sci. Eng. 54(1-2), 55-69 (2006). ISSN 0920-4105 
Dullien, F.A.: Porous Media: Fluid Transport and Pore Structure. Academic Press, London (2012)

Emami-Meybodi, H., Hassanzadeh, H., Green, C.P., Ennis-King, J.: Convective dissolution of co2 in saline aquifers: progress in modeling and experiments. Int. J. Greenh. Gas Control 40, 238-266 (2015)

Erfani, H., Joekar-Niasar, V., Farajzadeh, R.: Impact of microheterogeneity on upscaling reactive transport in geothermal energy. ACS Earth Space Chem. (2019)

Erfani, H., Babaei, M., Niasar, V.: Signature of geochemistry on density-driven $\mathrm{CO}_{2}$ mixing in sandstone aquifers. Water Resour. Res. 56(3), e2019WR026060 (2020)

Erfani Gahrooei, H., Ghazanfari, M.H.: Toward a hydrocarbon-basedchemical for wettability alteration of reservoir rocks to gaswetting condition: Implications to gas condensate reservoirs. J. Mol. Liq. 248, 100-111 (2017)

Erfani Gahrooei, H., Ghazanfari, M.H.: Wettability alteration of reservoir rocks to gas wetting condition: a comparative study. Can. J. Chem. Eng. 96(4), 997-1004 (2018)

Erfani Gahrooei, H., Joonaki, E.: A new empirical scaling equation for accurate prediction of gaseous species equilibrium adsorption on activated carbon. Asia-Pac. J. Chem. Eng. 13(4), e2220 (2018)

Ershadnia, R., Wallace, C.D., Soltanian, M.R.: $\mathrm{Co}_{2}$ geological sequestration in heterogeneous binary media: effects of geological and operational conditions. Adv. Geo-Energy Res. 4(4), 392-405 (2020)

Farajzadeh, R., Wassing, B., Boerrigter, P.M.: Foam assisted gas-oil gravity drainage in naturally-fractured reservoirs. J. Petrol. Sci. Eng. 94, 112-122 (2012). ISSN 0920-4105

Firoozabadi, A., Ishimoto, K.: Reinfiltration in fractured porous media: part 1-one dimensional model. SPE Adv. Technol. Ser. 2(02), 35-44 (1994). ISSN 1076-0148

Firoozabadi, A., Markeset, T.: Laboratory study of reinfiltration for gas-liquid system in fractured porous media (1992)

Firoozabadi, A., Markeset, T.: An experimental study of the gas-liquid transmissibility in fractured porous media. SPE Reserv. Eng. 9(03), 201-207 (1994). ISSN 0885-9248

Ghezzehei, T.A., Or, D.: Liquid fragmentation and intermittent flow regimes in unsaturated fractured media. Water Resour. Res. 41(12), W12406 (2005)

Gleeson, T., Novakowski, K., Kyser, T.K.: Extremely rapid and localized recharge to a fractured rock aquifer. J. Hydrol. 376(3-4), 496-509 (2009). ISSN 0022-1694

Hagoort, J.: Oil recovery by gravity drainage. Soc. Petrol. Eng. J. 20(03), 139-150 (1980)

Hakimov, N., Zolfaghari, A., Dahaghi, A.K., Negahban, S., Gunter, G.: Pore-scale network modeling of microporosity in low-resistivity pay zones of carbonate reservoir. J. Nat. Gas Sci. Eng. 71, 103005 (2019)

Harimi, B., Masihi, M., Ghazanfari, M.H.: Characterization of liquid bridge in gas/oil gravity drainage in fractured reservoirs. Iran. J. Oil Sci. Gas Technol. 8(2), 73-91 (2019). ISSN 2345-2412

Hasan, S., Joekar-Niasar, V., Karadimitriou, N.K., Sahimi, M.: Saturation dependence of non-Fickian transport in porous media. Water Resour. Res. 55(2), 1153-1166 (2019)

Hasan, S., Niasar, V., Karadimitriou, N.K., Godinho, J.R., Vo, N.T., An, S., Rabbani, A., Steeb, H.: Direct characterization of solute transport in unsaturated porous media using fast X-ray synchrotron microtomography. Proc. Nat. Acad. Sci. 117(38), 23443-23449 (2020)

Joonaki, E., Erfani Gahrooei, H., Ghanaatian, S.: Experimental study on adsorption and wettability alteration aspects of a new chemical using for enhanced oil recovery in carbonate oil reservoirs. $\mathbf{J}$. Unconv. Oil Gas Resour. 15, 11-21 (2016)

Khorshidian, H., James, L.A., Butt, S.D.: Demonstrating the effect of hydraulic continuity of the wetting phase on the performance of pore network micromodels during gas assisted gravity drainage. J. Petrol. Sci. Eng. 165, 375-387 (2018). ISSN 0920-4105

Kralchevsky, P., Nagayama, K.: Particles at Fluid Interfaces and Membranes: Attachment of Colloid Particles and Proteins to Interfaces and Formation of Two-Dimensional Arrays. Elsevier, Amsterdam (2001)

Leverett, M.: Capillary behavior in porous solids. Trans. AIME 142(01), 152-169 (1941). ISSN 0081-1696

Li, K., Horne, R.N.: Prediction of oil production by gravity drainage. In: SPE Annual Technical Conference and Exhibition. Society of Petroleum Engineers (2003)

Mashayekhizadeh, V., Ghazanfari, M.H., Kharrat, R., Dejam, M.: Pore-level observation of free gravity drainage of oil in fractured porous media. Transp. Porous Media 87(2), 561-584 (2011). ISSN 0169-3913

Moon, A., Bruce, A.F.: Flow of water in partially-saturated soils (2007)

Mualem, Y.: A new model for predicting the hydraulic conductivity of unsaturated porous media. Water Resour. Res. 12(3), 513-522 (1976). ISSN 1944-7973

Multiphysics, C.: Comsol multiphysics user guide (version 4.3 a). COMSOL, AB, pp. 39-40 (2012) 
Nenniger, E., Storrow, J.A.: Drainage of packed beds in gravitational and centrifugal-force fields. AIChE J. 4(3), 305-316 (1958). ISSN 1547-5905

Pavone, D., Bruzzi, P., Verre, R.: Gravity drainage at low ift. In Gravity drainage at low IFT. 5th Eur. Symp. Enhanced Oil Recovery, Budapest (1989)

Rabbani, A., Babaei, M., Javadpour, F.: A triple pore network model (t-pnm) for gas flow simulation in fractured, micro-porous and meso-porous media. Transp. Porous Media 2020, 1-34 (2020a)

Rabbani, A., Babaei, M., Shams, R., Da Wang, Y., Chung, T.: Deepore: a deep learning workflow for rapid and comprehensive characterization of porous materials. Adv. Water Resour. 2020, 103787 (2020b)

Rahmati, N., Rasaei, M.: Quantifying the reimbibition effect on the performance of gas-oil gravity drainage in fractured reservoirs: Mathematical modelling. Can. J. Chem. Eng. 97, 1718-1728 (2019). ISSN 0008-4034

Rokhforouz, M., Akhlaghi Amiri, H.: Phase-field simulation of counter-current spontaneous imbibition in a fractured heterogeneous porous medium. Phys. Fluids 29(6), 062104 (2017)

Rokhforouz, M., Amiri, H.A.: Pore-level influence of micro-fracture parameters on visco-capillary behavior of two-phase displacements in porous media. Adv. Water Resour. 113, 260-271 (2018)

Rokhforouz, M., Amiri, H.A.: Effects of grain size and shape distribution on pore-scale numerical simulation of two-phase flow in a heterogeneous porous medium. Adv. Water Resour. 124, 84-95 (2019). ISSN 0309-1708

Rostami, B., Kharrat, R., Pooladi-Darvish, M., Ghotbi, C.: Identification of fluid dynamics in forced gravity drainage using dimensionless groups. Transp. Porous Media 83(3), 725-740 (2010). ISSN 0169-3913

Saedi, B., Ayatollahi, S., Masihi, M.: Free fall and controlled gravity drainage processes in fractured porous media: laboratory and modelling investigation. Can. J. Chem. Eng. 93(12), 2286-2297 (2015). ISSN 1939-019X

Sahimi, M.: Flow and Transport in Porous Media and Fractured Rock: from Classical Methods to Modern Approaches. Wiley, New York (2011). ISBN 3527636706

Saidi, A.: Simulation of naturally fractured reservoirs. Society of Petroleum Engineers. In: SPE Reservoir Simulation Symposium (1983)

Saidi, A., Tehrani, D., Wit, K.: Mathematical simulation of fractured reservoir performance, based on physical model experiments, paper wpc18227. In: 10th World Petroleum Congress, pp. 9-14 (1979)

Sajadian, V., Danesh, A., Tehrani, D.: Laboratory studies of gravity drainage mechanism in fractured carbonate reservoir-capillary continuity. In Abu Dhabi International Petroleum Exhibition and Conference. Society of Petroleum Engineers (1998)

Schechter, D., Guo, B.: Mathematical modeling of gravity drainage after gas injection into fractured reservoirs. In Permian Basin Oil and Gas Recovery Conference. Society of Petroleum Engineers (1996)

Sebben, M.L., Werner, A.D., Graf, T.: Seawater intrusion in fractured coastal aquifers: a preliminary numerical investigation using a fractured Henry problem. Adv. Water Resour. 85, 93-108 (2015). ISSN 0309-1708

Soltanian, M.R., Hajirezaie, S., Hosseini, S.A., Dashtian, H., Amooie, M.A., Meyal, A., Ershadnia, R., Ampomah, W., Islam, A., Zhang, X.: Multicomponent reactive transport of carbon dioxide in fluvial heterogeneous aquifers. J. Nat. Gas Sci. Eng. 65, 212-223 (2019)

Stars, C., Guide, U.: Advanced Process and Thermal Reservoir Simulator. Computer Modelling Group Ltd, Calgary (2008)

Teng, Y., Liu, Y., Jiang, L., Song, Y., Zhao, J., Zhang, Y., Wang, D.: A visualization study on two-phase gravity drainage in porous media by using magnetic resonance imaging. Magn. Reson. Imaging 34(7), 855-863 (2016). ISSN 0730-725X

Udoh, F.D., Igbafe, A.I., Okon, A.N.: Gas-oil gravity drainage and reinfiltration in naturally fractured reservoirs. In: SPE Nigeria Annual International Conference and Exhibition. Society of Petroleum Engineers (2015)

Van Genuchten, M.T.: A closed-form equation for predicting the hydraulic conductivity of unsaturated soils. Soil Sci. Soc. Am. J. 44(5), 892-898 (1980). ISSN 0361-5995

Weisbrod, N., Dragila, M.: Potential impact of convective fracture venting on salt-crust buildup and groundwater salinization in arid environments. J. Arid Environ. 65(3), 386-399 (2006). ISSN 0140-1963

Zendehboudi, S., Chatzis, I., Shafiei, A., Dusseault, M.B.: Empirical modeling of gravity drainage in fractured porous media. Energy Fuels 25(3), 1229-1241 (2011). ISSN 0887-0624

Zendehboudi, S., Shafiei, A., Chatzis, I., Dusseault, M.B.: Numerical simulation of free fall and controlled gravity drainage processes in porous media. J. Porous Media 15(3), 2012 (2012)

Zobeidi, K., Fassihi, M.: Block to block interactions and their effects on miscibility gravity drainage in fractured carbonate reservoirs, experimental and analytical results. J. Petrol. Sci. Eng. 164, 696-708 (2018). ISSN 0920-4105 
Zobeidi, K., Fassihi, M.R., Rasaei, M.R.: Description of the results of experiments on developed miscibility with nonequilibrium gas/oil gravity drainage. SPE J. 21(03), 827-838 (2016). ISSN 1086-055X

Publisher's Note Springer Nature remains neutral with regard to jurisdictional claims in published maps and institutional affiliations.

\section{Affiliations}

\section{Hamidreza Erfani ${ }^{1,2}$ - Abtin Karimi Malekabadi ${ }^{3}$ Mohammad Hossein Ghazanfari ${ }^{2}$. Behzad Rostami ${ }^{4}$ (D)}

1 Department of Chemical Engineering and Analytical Science, University of Manchester, Oxford Road, Manchester M13 9PL, UK

2 Department of Chemical and Petroleum Engineering, Sharif University of Technology, Tehran, Iran

3 Ahwaz Faculty of Petroleum Engineering, Petroleum University of Technology, Ahwaz, Iran

4 Institute of Petroleum Engineering, School of Chemical Engineering, College of Engineering, University of Tehran, Tehran, Iran 